\title{
Short-Term Electricity Price Forecasting Based on Grey Prediction GM(1,3) and Wavelet Neural Network
}

\author{
Dong Jun, Yang Peiwen \\ Department of Economics and Management, North China Electric Power University, Beijing, China \\ Email address: \\ dongjun624@126.com (Dong Jun),965047290@qq.com (Yang Peiwen)
}

To cite this article:

Dong Jun, Yang Peiwen. Short-Term Electricity Price Forecasting Based on Grey Prediction GM(1,3) and Wavelet Neural Network. American Journal of Electrical Power and Energy Systems. Vol. 7, No. 4, 2018, pp. 50-55. doi: 10.11648/j.epes.20180704.12

Received: July 30, 2018; Accepted: August 13, 2018; Published: September 4, 2018

\begin{abstract}
Electricity price is a core index that reflects the operation status of the power market, evaluates the efficiency of market competition, and is the basis for decision-making in the electricity market. Electricity price forecasting is of great significance to guide investment, allocate market resources spontaneously, achieve a basic balance of power supply and demand, and meet various service goals. In this paper, a short-term electricity price forecasting method based on a grey forecasting $\operatorname{GM}(1,3)$ and wavelet neural network combination model is adopted. Firstly, the power price sequence is decomposed and reconstructed by using the famous MALLAT algorithm of multi-resolution analysis based on wavelet transform theory, and then the final predictive electricity price sequence is obtained by using the BP neural network model. Then the predicted electricity price sequence is used as a relevant factor affecting the future daily electricity price and input to the grey $\operatorname{GM}(1,3)$ forecasting model for electricity price forecasting to obtain the final forecasting result. The model training and forecasting based on the 2012 load and price data published by the PJM power market in the United States show that the prediction model established by this method has higher prediction accuracy. Thus, it has important research significance for electricity market price forecasting.
\end{abstract}

Keywords: Grey Prediction, Wavelet Neural Network, Electricity Price Forecasting Combination Model

\section{Introduction}

With the rapid advancement of China's new round of power reforms, power will resume its commodity attributes in the future. As the most important basic factor in the electricity market, electricity prices directly determine the returns of market participants. Therefore, it is especially important to judge the future electricity price trend. In other words, electricity price forecasting has far-reaching significance for market participants' investment decisions and risk management. The current electricity price forecast is divided into medium and long-term electricity price forecasting and short-term electricity price forecasting. The medium and long-term electricity price forecast is mainly based on electricity market trading rules to predict electricity prices through the simulation settlement process. The main methods include oligopolistic equilibrium model, random production simulation and intelligent simulation model. The size of short-term load forecast mainly depends on the prediction accuracy. Therefore, how to improve the prediction accuracy is the focus of current short-term load forecasting theories and methods. Short-term load forecasting has a long history. Many experts and scholars at home and abroad have conducted extensive research on prediction theory and methods, and have put forward many prediction models. By collecting and organizing a large amount of historical data, using statistical analysis methods, according to the trend of changes in electricity prices, and then select the appropriate mathematical model for prediction, through the literature can be found more research methods, such as: time series, neural networks, gray Prediction, dynamic econometrics, etc. [1-3].

From the related literature on electricity price forecasting, it can be found that the electricity price has the same characteristics as the load, but the electricity price also has many own characteristics, such as real-time balance, non-stationary, multi-periodic, mean resilience, strong volatility, etc. It is not only related to historical data, but it is also affected by many factors such as: load, supply and demand, temperature, time period, human factors, market 
system and structure, etc., all of which have a great impact on electricity prices. In particular, the influence of uncertainties (such as supply and demand changes, fuel price fluctuations, component breaks, network congestion, and market forces) makes accurate forecasting of electricity prices more difficult [4]. The short-term electricity price models are based on these main influencing factors of electricity prices, establishing a relationship model between these factors and electricity prices to minimize other interferences. Therefore, short-term electricity price forecasting should be devoted to tapping the characteristics of electricity price trend changes and establishing the best forecast through the combination of algorithm models. Model [5].

Short-term electricity price forecasting requires most historical data to support most models [6]. The grey forecasting model does not require the probability distribution of the original data series. It can achieve a small amount of data modeling and prediction, can adapt to the rapid changing behavior of the system, and the calculation is fast [7]. The model is relatively simple. Among them, $\operatorname{GM}(1,3)$ has improved the $\operatorname{GM}(1,1)$ model and introduced the electricity price related influencing factors, such as: historical electricity price, load curve, etc., making the model more reasonable[8]. However, selecting the original sequence of related factors often can not make the prediction of electricity price achieve a good prediction effect. Therefore, this kind of nonlinear and adaptive information processing system is adopted by wavelet neural network, and the relevant factor sequence is modified through network training to make the gray prediction model. Curve fitting is better [9]

This paper adopts the grey forecasting $\operatorname{GM}(1,3)$ model, selects two related factors ---historical electricity price and power load, and then uses the famous multi-resolution analysis MALLAT algorithm of wavelet transform theory to realize the decomposition and reconstruction of the electricity price sequence[10]. Selecting the appropriate wavelet basis and decomposition scale, the power price sequence is decomposed into a low-frequency component and multiple high-frequency components, so as to extract the price characteristics [11]. Using the obtained components of the electricity price, the BP neural network model is established separately for each component of the electricity price. Finally, the prediction results of the components are summed to obtain the final predicted electricity price sequence. Then the predicted electricity price sequence is used as the relevant factor affecting the future daily electricity price. It is input into the grey $\operatorname{GM}(1,3)$ forecasting model to predict the electricity price, and the final forecast result is obtained. Compared with the grey prediction model and the wavelet neural network model alone, the GM is used [12]. $(1,3)$ and wavelet neural network combined short-term electricity price forecasting model, its prediction accuracy is greatly improved, and has certain reference value and significance.

\section{Grey Prediction GM(1,3) Model}

The "gray system" theory was founded by Professor Deng
Julong in 1982. Gray prediction has the advantages of less original information needed, simple calculation process, predictable testability, etc. Therefore, it is widely used. The basic method of the gray model is described below.

The system characteristic data sequence,

$$
x_{1}^{(0)}=\left\{x_{1}^{(0)}(1), x_{1}^{(0)}(2), x_{1}^{(0)}(3), \ldots x_{1}^{(0)}(n)\right\}
$$

For two relevant factor sequences,

$$
\begin{aligned}
& x_{2}^{(0)}=\left\{x_{2}^{(0)}(1), x_{2}^{(0)}(2), x_{2}^{(0)}(3), \ldots x_{2}^{(0)}(n)\right\} \\
& x_{3}^{(0)}=\left\{x_{3}^{(0)}(1), x_{3}^{(0)}(2), x_{3}^{(0)}(3), \ldots x_{3}^{(0)}(n)\right\}
\end{aligned}
$$

(1) Ash generation

There are three types of generation methods commonly used in gray systems: Accumulated Generation (AGO), Reduced Generation (IAGO), and Map Generation. Only cumulative generation is considered here. That is, the original sequence and the related factor sequence are respectively accumulated and generated (AGO) to form a new cumulative sequence. Accumulate the original sequence and related factors, respectively, forms a new cumulative sequence:

$$
\begin{gathered}
x_{1}^{(1)}(k)=\sum_{j=1}^{k} x_{1}^{(0)}(j) \\
x_{1}^{(1)}=\left\{x_{1}^{(1)}(1), x_{1}^{(1)}(2), x_{1}^{(1)}(3), \ldots x_{1}^{(1)}(n)\right\} \\
x_{2}^{(1)}=\left\{x_{2}^{(1)}(1), x_{2}^{(1)}(2), x_{2}^{(1)}(3), \ldots x_{2}^{(1)}(n)\right\} \\
x_{3}^{(1)}=\left\{x_{3}^{(1)}(1), x_{3}^{(1)}(2), x_{3}^{(1)}(3), \ldots x_{3}^{(1)}(n)\right\}
\end{gathered}
$$

(2) The raw data series is averaged:

$$
Z_{k}=\frac{\left(x_{1}^{(1)}(k)+x_{1}^{(1)}(k-1)\right)}{2}(k=1,2,3 \ldots . n)
$$

(3) Similar to the GM $(1,1)$ model, constructing first-order linear differential equations is:

$$
\begin{aligned}
& \frac{d x_{1}^{(1)}}{d t}+a x_{1}^{(1)}=b x_{2}^{(1)}+b x_{3}^{(1)} \\
& \frac{d x_{2}^{(1)}}{d t}+a x_{2}^{(1)}=b x_{2}^{(1)}+b x_{3}^{(1)} \\
& \frac{d x_{3}^{(1)}}{d t}+a x_{3}^{(1)}=b x_{2}^{(1)}+b x_{3}^{(1)}
\end{aligned}
$$

This is a first-order, three-variable differential equation model, so it is denoted as $\operatorname{GM}(1,3)$. Remember that the parameters of the above equation are listed as $\hat{a}$, where $\hat{a}=$ $\left(\hat{a}+\widehat{b_{1}}+\widehat{b_{2}}\right)$, then $\hat{a}$ is obtained according to the least-squares method.

$$
\hat{a}=\left(B^{T} B\right)^{-1} B^{T} Y_{n}
$$

Among them 


$$
\mathrm{YN}=\left[\begin{array}{c}
x_{1}^{(0)}(2) \\
x_{1}^{(0)}(3) \\
x_{1}^{(0)}(4) \\
\cdot \\
\cdot \\
x_{1}^{(0)}(n)
\end{array}\right] \mathrm{B}=\left[\begin{array}{c}
-Z_{2} x_{1}^{(1)}(2) x_{2}^{(1)}(2) \\
-Z_{3} x_{1}^{(1)}(3) x_{2}^{(1)}(3) \\
-Z_{4} x_{1}^{(1)}(4) x_{2}^{(1)}(4) \\
\cdot \\
\cdot \\
-Z_{n} x_{1}^{(1)}(n) x_{2}^{(1)}(n)
\end{array}\right]
$$

From this, it can be found that the unique solution of the parameter column $\hat{a}$ substituting the obtained solution into the differential equation. In solving the equation:

$\widehat{x_{1}^{(1)}}(\mathrm{k}+1)=\left(x_{1}^{(0)}(1)-\frac{\widehat{b_{1}}}{\hat{a}} x_{2}^{(1)}(k+1)-\frac{\widehat{b_{2}}}{\hat{a}} x_{3}^{(1)}(k+1)\right) e^{-a t}$

$$
+\frac{\widehat{b_{1}}}{\hat{a}} x_{2}^{(1)}(k+1)+\frac{\widehat{b_{2}}}{\hat{a}} x_{3}^{(1)}(k+1)(k=1,2,3 \ldots n)
$$

(4) Calculate $x_{1}^{(0)}$ the estimated value $\widehat{x_{1}^{(0)}}$

$$
x_{1}^{\widehat{(0)}(k)}=x_{1}^{\widehat{(1)}(k)}-x_{1}^{(1)} \widehat{(k-1)}(k=2,3, \ldots n)
$$

\section{Wavelet Neural Network Model}

The wavelet neural network model flow diagram is as follows:

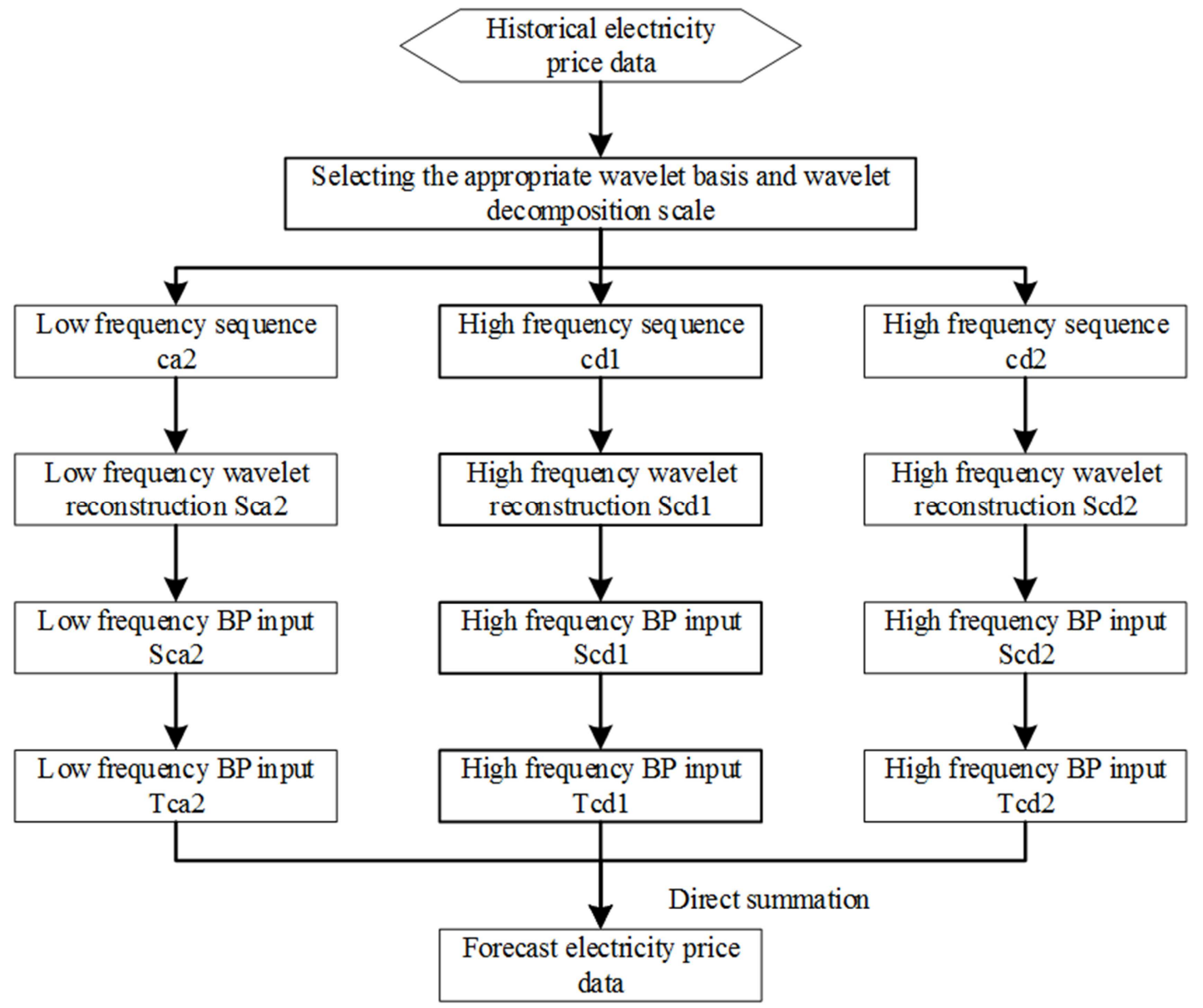

Figure 1. Flow diagram of neural network prediction model based on wavelet decomposition.

First, wavelet decomposition is used to remove the details of the original electricity price sequence and reconstruct the electricity price data. A large number of practices have shown that $\mathrm{dbN}$ wavelet analysis of time series has strong ability, and by comparing the decomposition sequence obtained by the corresponding functions of $\mathrm{N}, 1,2,3$, and 4 respectively, it is found that $\mathrm{db} 2$ as the wavelet base can best reflect the original electricity price sequence. The trend of change, therefore using $\mathrm{db} 2$ wavelet decomposition [13-14]. In order to maximize the prediction accuracy and reduce the cumulative error at the same time, scale 2 is selected as the wavelet decomposition scale. In order to accurately find out the variation rules of the large-scale electricity price sequence and the small-scale electricity price sequence, the following uses different BP neural network structures for each electricity price sub-sequence to predict. The correlation analysis 
technique was used to roughly determine the main influencing factors affecting each sequence. If $\mathrm{X}$ and $\mathrm{Y}$ are two vectors, the correlation coefficient $\mathrm{P} 2$ is used to describe the correlation between the vectors $\mathrm{X}$ and $\mathrm{Y}$. Its calculation formula is:

$$
\begin{gathered}
\rho_{x y}=\frac{\operatorname{cov}(X, Y)}{\sqrt{D(X) D(Y)}} \\
-1 \leq \rho_{x y} \leq 1 \\
\operatorname{cov}(X, Y)=\frac{1}{n} \sum_{i=1}^{n}\left(x_{i}-\mu_{x}\right)\left(y_{i}-\mu_{y}\right)
\end{gathered}
$$

$\rho_{x y}$ is used to characterize the closeness of the linear relationship between $\mathrm{X}$ and $\mathrm{Y}$. When $\rho_{x y}$ is larger, the degree of linear correlation between $\mathrm{X}$ and $\mathrm{Y}$ is better, and vice versa. By correlation analysis, it shows that the low-frequency part of the wavelet decomposition of the original sequence of the low-frequency part of the cat sequence has a certain periodicity, so choose the relevant prediction day, the day before the forecast and the previous two days before the forecast. Moreover, when the load and price data as input variables, and the original sequence of high-frequency wavelet decomposition components cdl and $\mathrm{cd} 2$ correlation are as follows: cdl has a short-term dependence, random changes. So select the first 12 moments. The price of electricity as an input variable; while cd2 has a large degree of volatility, but still shows a certain periodicity, and basically only affected by the price of electricity itself, so select the first time of the forecast, the first two days of the same time the price of electricity As input variable.

In addition, overfitting needs to be considered during the training of BP neural networks. Overfitting refers to the fact that although the error of the training set reaches the training target, when the new data is applied to the training network, the fitting error increases, that is to say, the neural network has memory for the training set, but for the new data Reduced generalization ability. Using the experimental method, first set a smaller number of neurons, train the network, calculate the approximation error, and then gradually increase the number of nodes until the approximation error is no longer significantly reduced.

After the wavelet transform, neural network prediction models with different connection weights and thresholds were established for sample data of different sequences. Sequences cat, cdl, and cd2 all use three-layer BP neural networks (network structures: 12-9-1, 12-8-1, and 24-16-D, respectively, for samples with non-structural and inaccurate patterns. Data is learned and trained, and complex nonlinear mappings from sample data to prediction data are completed.

\section{Analysis of Examples}

\subsection{Model Establishment}

According to the previous discussion of the method, the structural process of establishing a short-term electricity price forecasting model based on $\mathrm{WNN}-\mathrm{GM}(1,3)$ is shown in Figure 2.

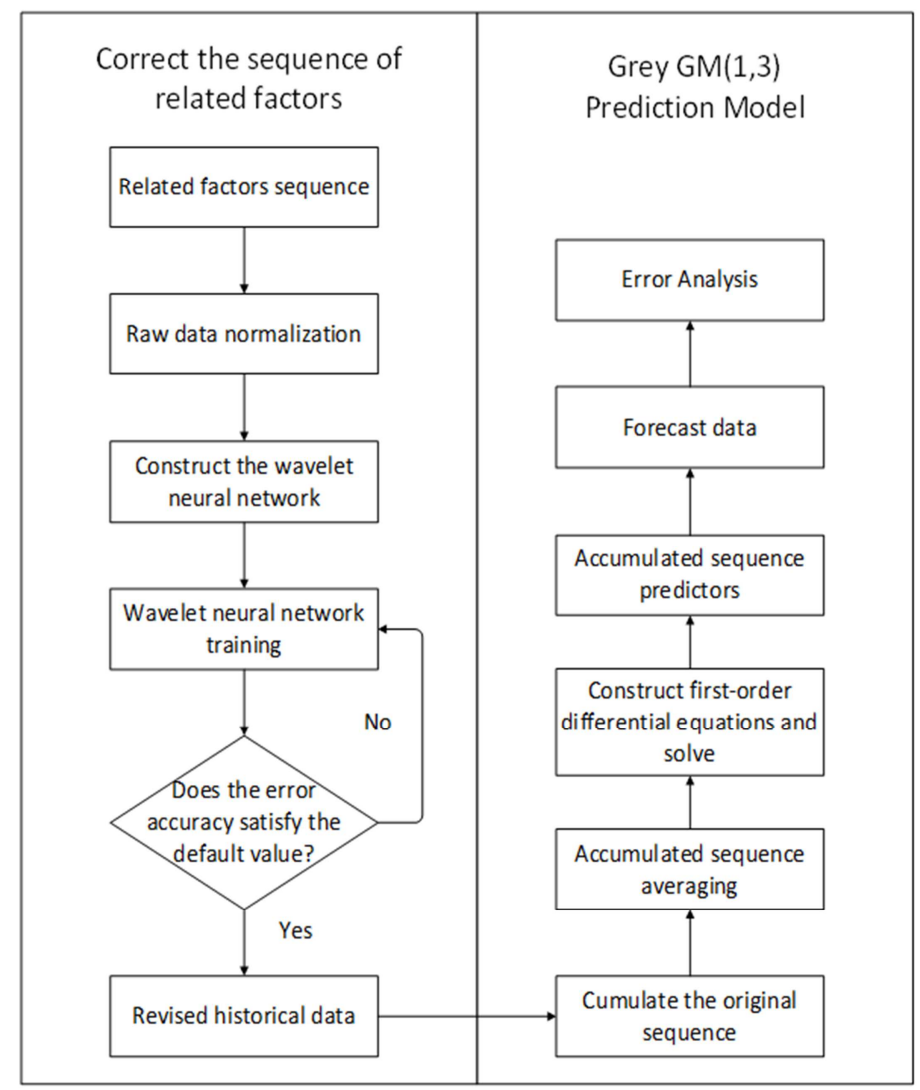

Figure 2. Structure of the short-term electricity price forecasting model based on $W N N-G M(1,3)$. 
The figure 2 shows that, first of all, select the relevant factors of historical electricity price and power load, and then use the famous multi-resolution analysis MALLAT algorithm of wavelet transform theory to achieve the decomposition and reconstruction of the electricity price sequence. Selecting the appropriate wavelet basis and decomposition scale, the power price sequence is decomposed into a low-frequency component and a plurality of high-frequency components, so as to extract the price characteristics. Using the obtained electricity price components, the BP neural network model is established separately for each component of the electricity price. Finally, the forecasting electricity price sequence is obtained by superimposing and superimposing the component prediction results. Then the predicted electricity price sequence is used as a correlative factor to influence the future daily electricity price. It is input into the grey $\operatorname{GM}(1,3)$ forecasting model to predict the electricity price. The original sequence is first accumulated, then averaged, and the first-order differential equation is constructed. Then reduce the reduction to get the final prediction result, and verify the accuracy of model prediction through error test.

\subsection{Sample Selection and Processing}

In order to verify the feasibility of the above methods, the historical operating data of the US PJM power market, which has relatively mature power market and regular electricity prices, was selected as a sample for simulation and prediction.

The node electricity price of the spot market from February
6, 2012 to February 12, 2012 is selected as the original electricity price sequence. To test the prediction effect, compare it with the other two models. The first kind of model directly uses $\operatorname{GM}(1,3)$ to predict the original electricity price and load sequence, and it is referred to as Model 1 for short. The second kind uses neural network model to predict electricity price (abbreviated as Model 2) directly. The third model uses the modified wavelet neural network for the original correlation factor sequence, and $\operatorname{GM}(1,3)$ prediction model (abbreviated as model 3) was used.

The final forecast results, using the error indicator $\delta_{\mathrm{MAPE}}$ as an evaluation of model performance, $\delta_{\text {MAPE }}$ is defined as follows:

$$
\left\{\begin{array}{l}
\delta_{\mathrm{MAPE}}=\frac{1}{N} \sum_{h=1}^{N} \frac{\left|\hat{p}_{h}-p_{h}\right|}{\bar{p}} \\
\bar{p}=\frac{1}{N} \sum_{h=1}^{N} p_{h}
\end{array}\right.
$$

In the formula, $\hat{p}_{h}, p_{h}$ are the forecasted electricity price and the actual electricity price respectively, and $\bar{p}$ means the average electricity prices during the forecast period, $N$ is the forecasting time points.

Figure 3 below shows the comparison of the original electricity price sequence with the prediction results of each model.

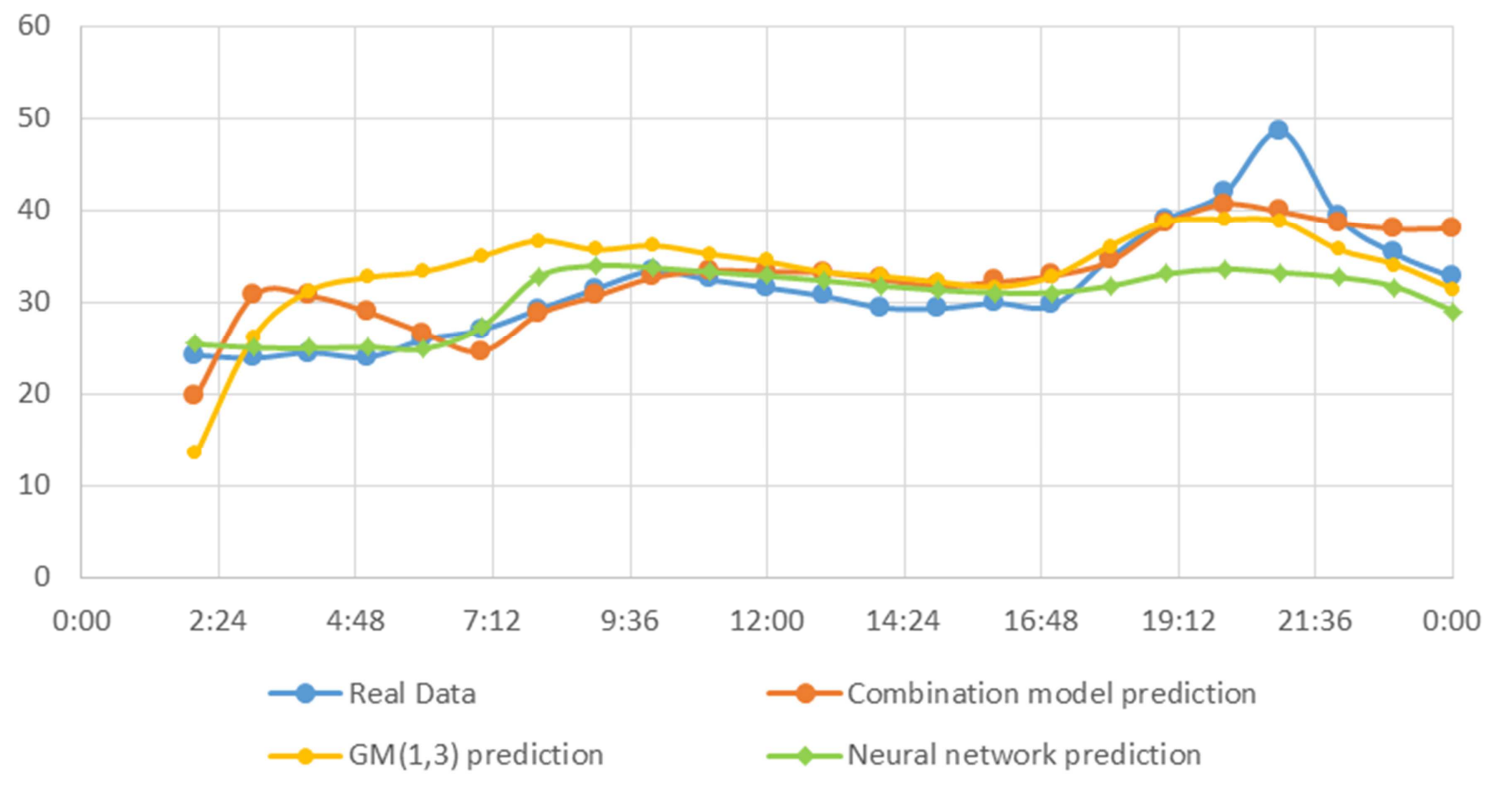

Figure 3. Original sequence and model prediction sequence.

From the above figure, it can be seen roughly that the prediction curve of the combined model is more fitted to the original data than the $\operatorname{GM}(1,3)$ prediction model and the neural network prediction model.

Then for the prediction data results, make an error test. The results are shown in Table 1 below.
Table 1. Comparison of model error test.

\begin{tabular}{llll}
\hline Number & Model 1 & Model 2 & Model 3 \\
\hline 1 & $34.37 \%$ & $2.69 \%$ & $14.17 \%$ \\
2 & $7.18 \%$ & $3.46 \%$ & $21.47 \%$ \\
3 & $21.54 \%$ & $1.93 \%$ & $19.66 \%$ \\
4 & $27.63 \%$ & $2.98 \%$ & $15.76 \%$ \\
\hline
\end{tabular}




\begin{tabular}{llll}
\hline Number & Model 1 & Model 2 & Model 3 \\
\hline 5 & $23.42 \%$ & $4.26 \%$ & $2.07 \%$ \\
6 & $25.43 \%$ & $18.44 \%$ & $7.50 \%$ \\
7 & $23.71 \%$ & $15.07 \%$ & $1.60 \%$ \\
8 & $13.47 \%$ & $7.30 \%$ & $2.46 \%$ \\
9 & $8.77 \%$ & $0.39 \%$ & $2.33 \%$ \\
10 & $8.84 \%$ & $1.32 \%$ & $3.13 \%$ \\
11 & $8.97 \%$ & $2.42 \%$ & $5.46 \%$ \\
12 & $8.26 \%$ & $3.49 \%$ & $8.07 \%$ \\
13 & $10.82 \%$ & $6.03 \%$ & $9.67 \%$ \\
14 & $9.16 \%$ & $5.41 \%$ & $7.75 \%$ \\
15 & $5.58 \%$ & $3.60 \%$ & $7.21 \%$ \\
16 & $10.02 \%$ & $6.69 \%$ & $10.21 \%$ \\
17 & $4.21 \%$ & $5.42 \%$ & $1.18 \%$ \\
18 & $0.70 \%$ & $17.24 \%$ & $1.50 \%$ \\
19 & $9.06 \%$ & $27.45 \%$ & $3.77 \%$ \\
20 & $31.30 \%$ & $50.60 \%$ & $27.98 \%$ \\
21 & $11.14 \%$ & $24.05 \%$ & $1.95 \%$ \\
22 & $3.67 \%$ & $19.56 \%$ & $8.62 \%$ \\
23 & $3.92 \%$ & $22.50 \%$ & $16.88 \%$ \\
$\delta_{\text {MAPE }}$ & $13.53 \%$ & $10.58 \%$ & $8.71 \%$ \\
\hline
\end{tabular}

From the table, obviously, $\delta_{\mathrm{MAPE}}^{1}>\delta_{\mathrm{MAPE}}^{2}>\delta_{\mathrm{MAPE}}^{3}$, so the deviation rate of Model 1 is the largest and the deviation rate of Model 3 is the smallest. It can be seen that the use of GM (1, 3 ) or neural network prediction model alone, the prediction effect is not as good as GM $(1,3)$ and wavelet neural network combination of this model has high prediction accuracy, fitting degree is more appropriate.

Therefore, using this combined model to predict electricity prices has research value and significance.

\section{Conclusion}

Using the $\operatorname{GM}(1,3)$ grey forecasting model, taking into account the impact of historical electricity price and load on the price trend, based on the mathematical theory of grey theory, the information resource of electricity price data is fully exploited to achieve less data modeling.

Using $\operatorname{GM}(1,3)$ and wavelet neural network combined method to forecast and analyze the electricity price of PJM power market in the United States, the prediction process is simple, the operability is strong, the accuracy of the prediction result is high, and the engineering application value is great. At the same time, it can also be seen that for a time series such as electricity price affected by many uncertainties, the combined forecasting model can improve the prediction accuracy more than the single model.

By comparing the combination forecasting method with the single forecasting method, this paper proves that the combination forecasting method has higher forecasting accuracy. Furthermore, it is of great value and significance for the in-depth study of the short-term electricity price trend forecasting in the future electricity market.

\section{References}

[1] Cheng Yuye. Research on short-term power load forecast based on artificial neural network [D]. Zhejiang University, 2017.

[2] Zou Zhiguo. Research on multi-component signal analysis method based on empirical mode decomposition[D]. Harbin Institute of Technology, 2016.

[3] GUO Xinxin. Short-term electricity price combination prediction based on EEMD and wavelet neural network[J]. Journal of Chongqing Technology and Business University, 2016, 33(02):21-25.

[4] Wang Huizhong, Zhou Jia, Liu Yu. A review of short-term load forecasting methods for power systems [J]. Electric Automation, 2015, 37(01): 1-3+39.

[5] Shen Xiaoling. Analysis of Factors Affecting Short-Term Load Forecasting of Power System [J]. Science \& Technology Information, 2011(05): 773-774.

[6] Wang Ruiqing, Li Yuzeng. Short-term electricity price forecasting using particle swarm optimization $\operatorname{GM}(1,2)$ with error correction [J]. Power System Protection and Control, 2011, 39(13): 41-45+52.

[7] Su Juan, Du Songhuai. Grey forecasting model of $\operatorname{GM}(1,2)$ short-term spot electricity price [J]. Relay, 2006(01):46-49.

[8] Jiang Jinan. Short-term power load forecasting based on wavelet denoising and artificial neural network [D]. Guangxi University, 2013.

[9] Liu Yusheng. Application of PSO-optimized $\operatorname{GM}(1,1)$ power-neural network model in short-term electricity price forecast [D]. Lanzhou University, 2011.

[10] YAMIN H Y, SHAH I S, LI Z . Adaptive Short-term Electricity Price Forecasting Using Artificial Neural Networks in the Restructured Power Markets [J]. Electrical Power and Energy Systems, 2004, 26( 8): 571-581

[11] WU Xinghua, ZHOU Hui. Short-term electricity price forecast based on subtraction clustering and adaptive fuzzy neural network [J]. Power System Technology, 2007 (19): 69-73.

[12] Chen Sijie. Application of wavelet neural network in power system short-term electricity price forecast [D]. Zhejiang University, 2006.

[13] Wang Meng, Jing Zhibin, Sun Bing, Liang Zhenfei. Prediction of short-term market clearing price based on BP neural network [J]. China Electric Power Education, 2011(30):100-102.

[14] WU Z, HUANG N E. Ensemble Empirical Mode Decomposition: A Noise-assisted Data Analysis Method [J]. Advances in Adaptive Data Analysis, 2009(1): 1-41. 